\title{
EXPERIENCIAS Y ASPECTOS A CONSIDERAR PARA LA IMPLEMENTACIÓN DE UN SISTEMA DE INFORMACIÓN DE APOYO PARA EL APRENDIZAJE A LO LARGO DE LA VIDA EN CHILE
}

\section{Óscar Espinoza ${ }^{1}$, Luis Eduardo González ${ }^{2}$}

\author{
RESUMEN
}

El objetivo del presente estudio es identificar los requerimientos para establecer un sistema de información que apoye el aprendizaje a lo largo de la vida, basado en un diagnóstico de la disponibilidad de datos existente en el país, utilizando un modelo de análisis ad hoc. Este incluye las estrategias necesarias para lograr la formación continua, los mecanismos e instrumentos para llevar a cabo las estrategias, la sugerencia de aspectos a considerar en el diseño de un futuro sistema de información de apoyo integrado y los recursos requeridos. La propuesta consigna cuatro dimensiones: las etapas de la trayectoria educativo-laboral, los requerimientos de información de diferentes usuarios, los usuarios propiamente tal, y los organismos proveedores de información.

Palabras clave: aprendizaje a lo largo de la vida, formación continua, sistema de información, trayectorias, educación superior

\section{EXPERIENCES AND ASPECTS TO CONSIDER IN THE IMPLEMENTATION OF A SUPPORTING INFORMATION SYSTEM FOR A LONG-LASTING LEARNING IN CHILE}

\section{ABSTRACT}

This study is aimed to identify the requirements for the creation of an information system oriented to support long lasting learning in Chile based on a diagnosis of the information available in different sources. To this end, it has developed an analytical model including strategies needed to achieve long lasting learning, mechanisms and instruments to carry out those strategies, recommendations for the design of such an information system and resources needed. Recommendations organize the resources in four dimensions: i) stages of labour-educational paths, ii) information requirements by different users; iii) users themselves; and iv) organizations providing information.

Keywords: long lasting learning, continuing education, information system, itineraries, higher education

1 Director. Centro de Investigación en Educación, Universidad UCINF, Santiago, Chile.

2 Investigador asociado. Centro de Investigación en Educación, Universidad UCINF, Santiago, Chile.

Contacto: oespinoza@ucinf.cl 


\section{EXPERIENCIAS Y ASPECTOS A CONSIDERAR PARA LA IMPLEMENTACIÓN DE UN SISTEMA DE INFORMACIÓN DE APOYO PARA EL APRENDIZAJE A LO LARGO DE LA VIDA EN CHILE}

\section{Antecedentes}

\subsection{Consideraciones iniciales}

El Consejo Nacional de Innovación para la Competitividad (CNIC, 2008) propuso tres grandes líneas estratégicas que el país debe implementar en el área del capital humano para avanzar hacia la economía del conocimiento: i) progresar hacia un sistema de aprendizaje a lo largo de la vida; ii) asegurar calidad y pertinencia de la formación y capacitación; iii) aumentar la cobertura de formación y capacitación con foco en sectores de menores ingresos y técnicos.

El CNIC afirma que se requiere en Chile de un sistema articulado de aprendizaje a lo largo de la vida -incluyendo formación y capacitación de técnicos y profesionales- que facilite tránsitos sucesivos entre el trabajo y la adquisición de nuevos conocimientos y habilidades, basado en la definición y certificación de competencias laborales, y en sintonía con los avances internacionales y con los requerimientos del mercado laboral.

El presente estudio apoya el análisis de estos objetivos desde la perspectiva de implementar un sistema de información que permita orientar y retroalimentar a los distintos actores del sistema de formación permanente, todo ello con el fin de asegurar buenas decisiones y la correcta articulación entre los distintos componentes de este sistema tan complejo.

\subsection{Aprendizaje a lo largo de la vida}

El concepto de aprendizaje permanente o formación a lo largo de la vida (Long Life Learning) se ha discutido en los países desarrollados 
desde la década de 1970. En el Informe de la Comisión Internacional de Educación sobre la formación para el siglo XXI de UNESCO se dice que "la Comisión ha optado por designar este proceso de aprendizaje continuo, que abarca toda la existencia y se ajusta a las demandas de la sociedad, con el nombre de educación a lo largo de la vida" (...) "el concepto de educación a lo largo de la vida puede considerarse como la clave desde la cual focalizar toda la educación. La perspectiva que abre las puertas del siglo XXI, va más allá de la distinción entre formación académica, actualización y reciclaje, reconversión y promoción de adultos. Se concibe como condición de desarrollo continuo y armónico de la persona" (Delors, Mufti, Amagi, Carneiro, Chung, Geremek, Gorham, Kornhauser, Manley, Quero, Savané, Singh, Stavenhagen, Won Suhr, Nanzhao, 1996, p. 113). Esta definición se inserta en la convicción de que todo momento y situación puede ser una ocasión para aprender y desarrollar las capacidades del individuo (Pacheco et al., 2005).

El aprendizaje a lo largo de la vida -que adquiere cada vez mayor relevancia, particularmente en el contexto de la naciente sociedad del conocimiento, donde el capital humano representa un elemento crucial para el desarrollo- puede considerarse como una formación que comienza en la infancia y se prolonga durante la vida de la persona y cuyos objetivos trascienden la calificación profesional, aunque esta dimensión tenga una presencia fundamental, impregnando todas las dimensiones que pueden influir en el desarrollo integral de las personas (Bermejo, 2006).

Aunque se suelen utilizar indistintamente los términos de educación continua y formación a lo largo de la vida, la tendencia de los últimos años al interior del Consejo de Europa ha sido distinguir con el nombre de "formación continua" a aquella parte del proceso dirigida principalmente a la preparación, actualización y perfeccionamiento profesional en la etapa postescolar. El Banco Mundial ha desarrollado en paralelo el concepto de educación continua de adultos (Adult Continuing Education) como parte integral del aprendizaje permanente, que podría producir importantes beneficios individuales contribuyendo al desarrollo económico y de capital humano de los países, y que contempla la inserción de los 
adultos en las universidades para ofrecer oportunidades reales de aprendizaje adulto para todos (OCDE, 2003).

En todo caso, más allá de las diversas definiciones, es posible considerar algunos aspectos comunes para la mayoría de ellas (Pacheco et al., 2005; Zúñiga, 2005):

- La creciente globalización, los cambios experimentados por la sociedad y la consolidación de la economía del conocimiento exigen nuevos y dinámicos conocimientos y competencias, generan diferentes desafíos y exigen una redefinición y renovación de los objetivos de las políticas educativas, enfocándolas hacia el aprendizaje permanente y obligando a una mayor sintonía con las demandas del mercado laboral, especialmente en los países en desarrollo.

- El concepto de aprendizaje a lo largo de la vida redefine la idea de aprendizaje, entendiéndolo como un proceso de construcción de conocimiento. Va más allá de la educación y enseñanza formal, pues incorpora los aprendizajes desde la niñez a la vejez en diferentes entornos, tanto formales como informales.

- El nuevo enfoque plantea nuevos métodos de aprendizaje: e-learning y el uso de nuevas tecnologías de aprendizaje a distancia.

\section{Marco conceptual y modelo de análisis}

La masificación de la matrícula es uno de los rasgos distintivos de los sistemas educativos en el mundo. Como consecuencia de ello, cada año hay un mayor número de personas con un nivel de instrucción superior que buscan continuar su formación o ingresar al mercado laboral. Para facilitar este proceso, los países necesitan proveer información y crear dispositivos de orientación y apoyo para las decisiones vocacionales y profesionales. Los países desarrollados cuentan con sofisticados sistemas de información educacional y laboral para los jóvenes y adultos, con políticas que procuran responder al requerimiento de nuevas competencias y exigencias profesionales a través de procesos de aprendizaje a lo largo de la vida. Para ello se ha diseñado un modelo de análisis que incluye el objetivo central de este estudio, las estrategias necesarias para lograrlo, los mecanismos 
e instrumentos para llevarlas a cabo y la propuesta de un sistema de información de apoyo que incluye los recursos requeridos. En la tabla $n .^{\circ} 1$ se describe el modelo de análisis y cada uno de sus componentes.

\subsection{Objetivo}

El objetivo del presente estudio es identificar los requerimientos para establecer un sistema de información que apoye el aprendizaje a lo largo de la vida basado en un diagnóstico de la disponibilidad de la información existente en el país, utilizando para ello un modelo de análisis ad hoc.

Tabla n. ${ }^{\circ} 1$ Modelo para analizar fuentes de información existentes asociadas con el aprendizaje a lo largo de la vida

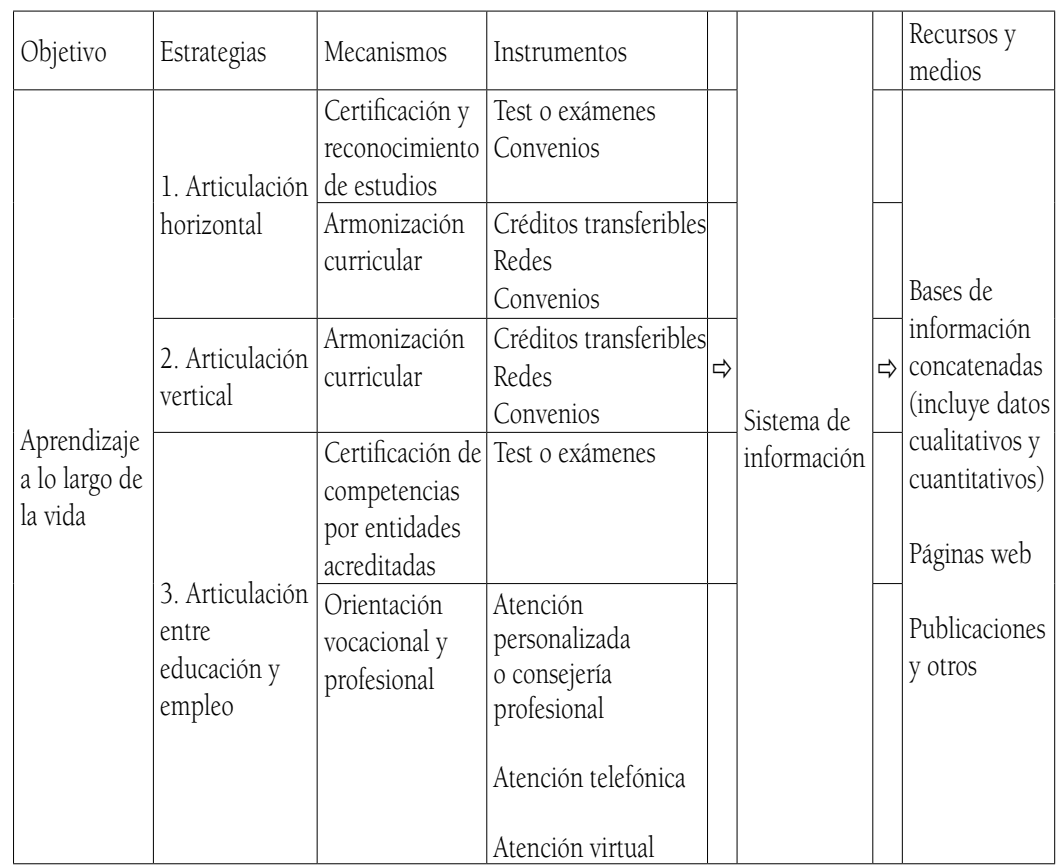

\subsection{Estrategias para fomentar el aprendizaje a lo largo de la vida \\ El capital humano está asociado a los individuos y su conocimiento, el cual se acumula y actualiza a través del tiempo mediante los}


aprendizajes que se puedan adquirir de diversas fuentes que incluyen la educación formal y la educación no formal. Con esta lógica, la educación continua está vinculada a los sistemas nacionales de calificación, al reconocimiento de aprendizajes previos, al currículo basado en competencias y al reconocimiento de carga por créditos transferibles (OCDE, 2007).

Para la conformación efectiva de los sistemas de formación de capital humano, desde la perspectiva de la educación permanente se plantean tres estrategias: i) la articulación horizontal; ii) la articulación vertical entre los distintos niveles de la educación formal y no formal; y iii) la articulación entre educación y empleo (CNIC, 2008).

\subsubsection{Articulación horizontal}

Esta estrategia se orienta a permitir equivalencias en los procesos de formación que faciliten la movilidad y el diseño de itinerarios en un mismo nivel por parte de los estudiantes de manera flexible. La articulación horizontal debe entenderse como una instancia de reconocimiento de la calidad y equivalencia de los estudios de un mismo nivel -por ejemplo, entre centros de formación técnica o entre universidades- entre todas las instituciones que componen los sistemas educativos, lo que supone el reconocimiento de estudios y asignaturas.

La articulación horizontal también supone un reconocimiento de los aprendizajes y las habilidades adquiridas tanto por la vía de estudios como a través de experiencia laboral, lo que supone contar con un proceso de certificación de competencias laborales que reconoce formalmente las capacidades y habilidades de un trabajador -sin importar cómo las adquirió- mediante una evaluación basada en criterios específicos, definidos mediante estándares validados por el mundo productivo y de acuerdo a una metodología preestablecida.

\subsubsection{Articulación vertical}

La articulación vertical se puede dar en dos ámbitos, usualmente en carácter ascendente: el de la continuidad de estudios formales y el de la transición entre la educación no formal y la formal. En relación 
con la progresión entre los distintos niveles de la educación formal -básica, media y superior-, la articulación ha de ser asimilada como el proceso de vinculación de dos o más calificaciones, a menudo obtenidas en diferentes niveles educativos, de modo que a través de certificaciones claramente definidas entre los distintos niveles se permita a los individuos avanzar en su trayectoria formativa. En lo que respecta a la transición entre la educación no formal -capacitación-y la formal, la articulación vertical permite a los individuos incrementar su nivel de escolaridad a través de la capacitación que se entrega fuera del sistema escolar y que es reconocida por éste.

En este ámbito cabe destacar que el fenómeno de la deserción es relevante particularmente en la educación media y, en menor medida, en la educación básica, aun cuando en comunas vulnerables es bastante significativo (Espinoza, González, Castillo y Loyola, 2011). Un estudio reciente (Latorre, González y Espinoza, 2009) concluye que en el caso de la cohorte de ingreso 1996 (primer año básico) apenas el 30\% logró concluir la enseñanza media en el tiempo oficial establecido y el 15\% aproximadamente ingresó a la educación superior. Junto con ello, se han hecho algunas estimaciones basadas en distintos modelos estadísticos para estimar la transición entre la educación media y la superior (Espinoza, González y Uribe, 2011). Con el fin de brindar alternativas a los jóvenes para que puedan culminar su enseñanza básica y media, se han establecido varias opciones. Una de esas modalidades es la de programas que permiten cursar dos o más niveles en un año académico. Otra es la de programas especiales conducentes a la obtención de licencia secundaria solo para fines laborales. Una tercera opción es la de reinserción laboral para adolescentes desertores.

\subsubsection{Articulación entre educación y empleo}

La formación para el trabajo se puede dar a través de tres vías: la educación formal, la educación no formal y la educación informal. La formal se realiza mediante la educación media técnico-profesional y en el nivel terciario se da a través de la formación de técnicos, profesionales y especializaciones en el nivel postsecundario. La no formal se da a través de diversas instancias de capacitación profesional. 
Y la informal es aquella que se da por la propia experiencia del desempeño laboral y por la interrelación con otros trabajadores.

Las dos primeras modalidades se orientan, generalmente, hacia el sector formal de la economía, esto es a empresas establecidas que ofrecen trabajo estable. No obstante, la mayor proporción del empleo se genera en el sector informal de la economía, así como en la microempresa (vale decir, en empresas de menos de cinco trabajadores, en unidades productivas familiares) y en trabajos independientes. En este contexto, se genera una disonancia entre la formación que se entrega y las demandas del sector productivo, ya que el área informal de la economía requiere de capacidades muy diferentes como son emprendimiento, creatividad, autogestión y polifuncionalidad. De ahí que los jóvenes perciban que no existe una ruta segura y definida para su inserción en el mundo laboral.

Como herramientas para la formación de jóvenes que pretenden integrarse al mundo laboral se han planteado, entre otras: i) facilitar el acceso juvenil al sistema regular de educación, introduciendo flexibilidad y objetivación curriculares; ii) aumentar la empleabilidad del joven mediante una educación que entregue las herramientas básicas para el desempeño laboral; iii) desarrollar, con el mismo objetivo, su familiaridad con el trabajo y la empresa; y iv) preparar especialistas en atención de jóvenes y en el tratamiento de los problemas propios de ese segmento.

\subsection{Mecanismos e instrumentos para implementar las estrategias}

Como se observa en la tabla $n .^{\circ} 1$, no hay una relación de correspondencia unívoca entre estrategias y mecanismos, ya que la mayoría de estos últimos son aplicables a cualquiera de las tres estrategias preestablecidas en el modelo.

Es posible distinguir, a lo menos, cuatro mecanismos que permiten implementar las distintas estrategias vinculables al aprendizaje a lo largo de la vida: i) certificación y reconocimiento de estudios; ii) armonización curricular que inevitablemente implica 
una readecuación o rediseño del currículo; iii) certificación de competencias por entidades acreditadas; y iv) orientación vocacional y profesional. Cabe insistir en que el mecanismo de armonización curricular puede estar asociado tanto a la articulación horizontal como vertical, e incluso con la articulación entre educación y empleo. Para efectos de este documento se optó por vincularlo preferentemente a la estrategia de articulación vertical dado que la experiencia de los casos estudiados aparece más enfatizada en este sentido. No obstante, cada mecanismo puede ser operacionalizado a través de uno o más instrumentos de distinta índole.

\subsubsection{Certificación y reconocimiento de estudios}

La certificación y el reconocimiento de estudios son la vía mediante la cual una institución educativa valida el trabajo académico realizado por un estudiante en otra entidad que le merece fe y confiabilidad. Una forma de garantizar la calidad de los estudios desarrollados en diversas instituciones es mediante procesos de acreditación con amplia validez, ya sea en el ámbito nacional o internacional, lo cual asegura el cumplimiento de estándares mínimos.

Existe un conjunto de términos que son asimilables, conceptualmente, en distintos lugares, tales como: reconocimiento, homologación, equiparación, equivalencia, revalidación, convalidación y certificación. No obstante, en esa diversidad se pueden distinguir distintos aspectos que dicen relación con la certificación, esto es: dar fe pública de la validez de estudios o los diplomas entregados, así como de los procedimientos para certificarlos. En este sentido, se puede reconocer, por una parte, la simple autentificación de los diplomas otorgados en otra institución atendiendo a su denominación original y, por otra, la homologación de diplomas o estudios en términos que habiliten para el ejercicio laboral o para proseguir estudios en un nivel superior (CINDA, 2000).

Para promover la certificación de estudios se pueden utilizar como instrumentos tanto los exámenes o test como los convenios que permiten una certificación automática. 


\subsubsection{Armonización curricular}

La armonización curricular para un sistema integrado de educación para toda la vida se orienta a permitir equivalencias en los procesos de formación que permitan la movilidad y el diseño de itinerarios flexibles por parte de los estudiantes.

Uno de los mayores niveles de armonización curricular se ha dado en la educación postsecundaria europea, que ha servido de modelo para Chile. En ella, se reconoce la cantidad de trabajo académico que realiza cada estudiante en función de sus propios intereses y su especialización por sobre los contenidos específicos de cada una de las asignaturas.

La armonización curricular se refleja en el avance paulatino del proceso de reforma de la educación superior europea, la cual se ha dado en seis frentes: i) reconocimiento de la carga académica expresada en créditos; ${ }^{3}$ ii) organización de los estudios en dos ciclos (bachiller o licenciado y magíster); iii) suplemento del diploma que individualiza y explicita las competencias del estudiante facilitando la interrelación con los empleadores; iv) títulos conjuntos, en particular para los estudios de doctorado organizados por asociaciones de universidades de varios países; v) acreditación y evaluación de la calidad de los programas; y vi) movilidad e intercambio estudiantil (Molitor, 2005).

Es importante señalar que para la formación de capital humano es necesario promover la interacción entre las distintas entidades públicas y privadas encargadas de formar y capacitar recursos, ya sea por la vía de la integración o cooperación. Esta última es un elemento fundamental para la reforma del sistema de educación superior, ya que fortalece la apertura y el impulso al trabajo conjunto y dinámico en todas direcciones y niveles, facilitando un mejor aprovechamiento de los recursos existentes y mejorando la calidad de los servicios educativos ofrecidos por las instituciones (Zúñiga, 2005).

Los instrumentos asociados a este mecanismo son los créditos transferibles, las redes y los convenios.

3 En el caso chileno el SCT-Chile fue adoptado por el CRUCH para incentivar la movilidad estudiantil (CRUCH, 2007). 


\subsubsection{Certificación de competencias por entidades acreditadas}

Los sistemas de certificación de competencias laborales y el aprendizaje a lo largo de la vida consideran el aprendizaje formal (programas conducentes a títulos y grados), el aprendizaje no formal (aprendizaje en actividades sistemáticas organizadas pero sin reconocimiento formal) y el aprendizaje informal (que se da en situaciones cotidianas de trabajo u otras) (Araneda, 2006; Behringer y Coles, 2003). La propuesta para transparentar los resultados de los procesos de aprendizaje para los empleadores y para las instituciones de formación es la medición de las competencias reales de las personas. Este enfoque se centra en la certificación de resultados y no se involucra en los procesos formativos, no obstante sus resultados retroalimentan la formación. Estos sistemas han estado orientados a las competencias laborales, con un carácter concreto y práctico. Son mecanismos paralelos a los procesos de armonización curricular.

En países anglosajones se ha utilizado el desarrollo e instalación de sistemas nacionales de competencias laborales como estrategia fundamental para articular las demandas del mercado laboral y los procesos de formación de capital humano (Cedefop, 2002). Los instrumentos vinculados a este mecanismo son los test o exámenes.

\subsubsection{Orientación vocacional y profesional}

Existen diferentes maneras de hacer que la orientación vocacional y profesional sea accesible a sectores más amplios de la población durante toda la vida y de responder a las necesidades de colectivos más diversos. Esto pasa por la reorganización del trabajo, la adopción de herramientas y nuevas técnicas y la inversión en nuevas tecnologías.

A partir del análisis de treintaiséis países, la OCDE (2003) estableció un conjunto de criterios para crear un sistema coherente de orientación profesional a lo largo de la vida, los que se enumeran a continuación: i) transparencia y fácil acceso a información relevante para la toma de decisiones; ii) dar importancia a los aspectos claves de la transición de un nivel educativo a otro, o bien en cuanto a la relación entre educación y trabajo; iii) un sistema de provisión innovativo y flexible que responda a diversas necesidades; iv) revisión 
y planificación sistemática; v) acceso a la orientación personalizada para aquellos que lo necesitan; vi) programas de desarrollo de capacidades de autogestión para los jóvenes; vii) oportunidades para explorar adecuadamente las opciones laborales antes de adoptar una decisión; viii) proveer información y consejería imparcial; ix) información integrada de educación y trabajo; y x) involucramiento activo de los beneficiarios.

Existen diversos instrumentos para desarrollar la orientación vocacional y profesional, a saber: la atención personalizada o consejería profesional presencial; la atención telefónica; y la atención virtual, que puede ser interacción vía electrónica con el interesado o bien con alguna comunidad virtual.

\subsection{Sistema de información}

Siguiendo con el modelo, todo lo que se ha señalado anteriormente está orientado a sistematizar los datos que alimenten un sistema de información que apoye el aprendizaje a lo largo de la vida, que constituya una herramienta de ayuda para la toma de decisiones de las personas y de las instituciones. En efecto, el sistema en cuestión permite concatenar datos de modo que tengan sentido para conocer una realidad determinada. Para ello, es necesario aunar información dispersa con formatos diferentes que no permiten una visión y un análisis global. Del mismo modo, se requiere evitar duplicaciones y hacer un mantenimiento para asegurar que los datos sean veraces y confiables.

Es indispensable contar con información de calidad que reúna las siguientes características:

- Homologable: que para todos los usuarios y proveedores signifique lo mismo.

- Coherente: que las variables guarden relación entre sí y que no aparezcan situaciones anómalas.

- Consistente: que el dato tenga un mismo valor para todas las instancias que entregan información.

- Oportuna: que se disponga en el momento que sea requerida por los usuarios. 
- Confiable: que sea entregada por fuentes responsables de su veracidad.

- Validada: que sea revisada para asegurar que no tenga errores.

- Actualizada: que se haga mantenimiento periódico y permanente para evitar errores.

La perspectiva de la educación permanente y los cambios en los sistemas de formación que ella acarrea, demandan también la existencia de un sistema de información capaz de orientar y retroalimentar a los distintos actores del sistema con el fin de asegurar buenas decisiones y la correcta articulación entre los distintos componentes de este sistema tan complejo.

Consecuentemente, un sistema de información adecuado para apoyar la formación permanente -considerando el diseño e implementación de políticas públicas asociadas con el desarrollo de capital humano- debiese proveer datos relativos a: i) oferta de formación disponible -programas, carreras, cursos, contenidos, tipo de certificación a la que conduce, acreditaciones y precios, entre otros- para que estudiantes, profesionales, trabajadores y empleadores del sector productivo puedan rentabilizar su inversión en materia formativa; ii) demanda de capital humano -nuevos nichos de empleo, sectores productivos y de servicios en expansión, perfiles laborales, entre otros- para los oferentes de formación y capacitación, con el objeto de fomentar una oferta pertinente y de calidad en sintonía con las necesidades y requerimientos del mercado laboral; y iii) mercado laboral y condiciones de empleabilidad para que estudiantes, profesionales y trabajadores puedan decidir, basados en información actualizada, los itinerarios formativos que respondan a sus expectativas laborales y vocacionales.

\subsection{Recursos y medios}

La información pública requiere un conjunto de recursos y medios, incluyendo las bases de información, las páginas web y las publicaciones. Existen diversos modelos entre los cuales se pueden citar las bases de datos independientes y autosoportantes, o bien las bases de datos concatenadas, en las cuales se recoge información 
desde fuentes diversas, la que se interrelaciona a través de elementos o variables comunes como el RUT.

\section{Experiencias e iniciativas desarrolladas en Chile en la perspectiva de la educación permanente}

En los párrafos siguientes se intenta dar una visión respecto de lo que acontece en Chile con la información disponible para apoyar la educación permanente. La sistematización de las iniciativas existentes se analiza utilizando el mismo esquema propuesto en el modelo anteriormente descrito. Como se podrá constatar, existen diversos espacios y canales de información, los cuales no se encuentran articulados entre sí.

Para comprender mejor el contexto, que sirve de base para operacionalizar el modelo de análisis, se caracteriza en primer lugar el sistema educativo chileno. Dicho sistema se organiza en cuatro niveles: preescolar, educación general básica, educación media -en dos modalidades: humanístico-científica y técnico-profesional (EMTP)- y educación superior. Existe la educación formal de adultos para los mayores de dieciocho años que no hayan completado sus estudios obligatorios. Esta opción educacional tiene alta relevancia, ya que según datos de la encuesta CASEN 2006 un 99,1\% de las personas de trece años acudía a un establecimiento educacional, a los diecisiete años el porcentaje bajaba al $85,2 \%$ y al $62 \%$ a los dieciocho años.

En la educación superior existen tres tipos de instituciones: las universidades (con carreras de cinco o más años), los institutos profesionales (con carreras de cuatro años) y los centros de formación técnica (con carreras de dos años). En 2004, para el nivel de pregrado se estimaba una cobertura total del $46 \%$ del grupo etario correspondiente ${ }^{4}$. Entre ellos, un 70\% estudiaba en carreras universitarias y el resto en institutos profesionales y centros de formación técnica (MINEDUC, 2009, 2009c).

$4 \quad 18$ a 24 años de edad. 
La educación no formal en Chile es responsabilidad del Servicio Nacional de Capacitación y Empleo (SENCE) y de las propias empresas (hasta el año 2009 también del Programa Chilecalifica). Esta se lleva a cabo a través de diversos programas que, en general, se implementan por medio de organismos técnicos de capacitación (OTEC), los cuales son certificados por el SENCE. El Estado apoya la capacitación mediante aportes directos del Ministerio del Trabajo (MINTRAB) y la reducción de impuestos a las empresas que capacitan a sus empleados. Asimismo, otros ministerios realizan diversas acciones de capacitación y mejoramiento de la calidad de vida mediante programas específicos.

De acuerdo con el modelo de análisis, el objetivo del aprendizaje a lo largo de la vida se logra mediante las estrategias de articulación horizontal, vertical y entre educación y empleo. Las estrategias concebidas en el modelo y asociadas al objetivo de la educación permanente definen los mecanismos de certificación de estudios y competencias, armonización curricular y orientación vocacional, las cuales deberían implementarse a través de distintos instrumentos. No obstante, para efectos de este documento solo se analizará el nivel de estrategias de apoyo para el aprendizaje permanente, obviándose los mecanismos e instrumentos asociados a ellas.

Todo lo anterior constituye el pilar fundamental para llevar adelante el análisis sobre el sistema de información de apoyo a la educación permanente existente en Chile. No obstante, en el caso chileno resulta complejo referirse a este debido a que existe una gran dispersión de iniciativas sin que exista una articulación entre ellas.

Una vez definido e identificado lo que podría ser un sistema de información se determinan los recursos que están disponibles para su implementación.

\subsection{Estrategias implementadas en Chile que podrían} contribuir a la generación de un sistema de información para la educación a lo largo de la vida

Siguiendo el modelo de análisis, las estrategias para dar sustento a un potencial sistema de información incluyen la articulación vertical, 
140 EXPERIENCIAS Y ASPECTOS A CONSIDERAR PARA LA IMPLEMENTACIÓN DE UN SISTEMA DE INFORMACIÓN DE APOYO PARA EL APRENDIZAJE A LO LARGO DE LA VIDA EN CHILE - O. Espinoza, L.E. González

horizontal y la articulación entre educación y empleo. A continuación se discute cada una de esas estrategias para el caso de Chile.

\subsubsection{Articulación horizontal}

La educación escolar en Chile tiene un sistema único de reconocimiento de las calificaciones, para lo cual existe un registro nacional dependiente del Ministerio de Educación. Este sistema permite la movilidad y el cambio de los estudiantes de un establecimiento a otro sin dificultades.

Los mayores de quince años que no asisten al colegio pueden rendir exámenes de validación de estudios en dos modalidades, una para continuar estudiando en algún nivel del sistema educativo, y otra sólo válida para efectos laborales (MINEDUC, 2009f).

En la educación superior, existen diversas y dispersas formas de reconocimiento de estudios parciales o de grados obtenidos, los cuales tienen distintas nomenclaturas: la convalidación de estudios parciales hechos en el extranjero, y la homologación de estudios, el "reconocimiento" propiamente tal, y la "revalidación". Tanto el reconocimiento de estudios como la revalidación solo pueden ser llevados a cabo por la Universidad de Chile.

Junto a estas formas de equivalencia de estudios existe un reconocimiento legal, producto de acuerdos internacionales, mediante el cual los grados o títulos otorgados en otros países tienen validez en Chile una vez hechos los trámites ante el Ministerio de Relaciones Exteriores (González, 2000; González y Ayarza, 1998).

Respecto de la movilidad entre instituciones de educación superior se destaca el esfuerzo que han hecho particularmente las Universidades del Consejo de Rectores (CRUCH) y algunas redes de carreras. En esta línea, las universidades del CRUCH definieron de mutuo acuerdo el Sistema de Créditos Transferibles-Chile (SCT-Chile) que permite el reconocimiento de estudios entre las diversas entidades (Ver CRUCH, 2007; Prieto y Mujica, 2007). 


\subsubsection{Articulación vertical}

El tránsito de la educación media a la superior exige la licencia secundaria. Para las universidades del CRUCH y algunas universidades privadas existe una prueba nacional unificada (Prueba de Selección Universitaria, PSU) como condición de ingreso.

Por su parte, el Programa Chilecalifica, mientras estuvo operando (fue discontinuado a fines del año 2009 por el gobierno de Michelle Bachelet), tuvo como propósito articular por la vía del reconocimiento de estudios las distintas instancias de educación técnica -tanto formal como no formal-, es decir, la capacitación -que realizaban las OTEC-, la educación media técnico profesional, la formación de técnicos superiores de centros de formación técnica, institutos profesionales y universidades, así como la vinculación con los organismos de capacitación, gestión y financiamiento en materia de preparación para el mundo del trabajo - como INDAP y SENCE-, avanzando en el establecimiento de un sistema de formación permanente. Además, este Programa generó oportunidades para adultos que querían completar su escolaridad mediante: educación regular de adultos, modalidad flexible de nivelación de estudios y la campaña de alfabetización "Contigo aprendo" (Chilecalifica, 2009a). El MINEDUC, a través de la Dirección General de Educación (DGE), creó una Coordinación de Adultos que sustenta estas últimas iniciativas.

En el ámbito de la educación terciaria, no existen canales transversales o nacionales oficiales para la articulación entre niveles de este subsistema, salvo algunos esfuerzos de articulación e integración vertical entre niveles formativos de una misma institución y que responden más bien a estrategias de retención de estudiantes.

Como una forma de completar la educación formal, Chilecalifica planteó como objetivo nivelar las habilidades transversales de los adultos, aspecto que fue asumido por el MINEDUC tras el cierre del programa. Para trabajadores adultos, las empresas pueden hacer uso de la franquicia tributaria que les permite recuperar impuestos hasta del 1\% del valor de sus planillas de sueldo con el fin de nivelar estudios básicos y medios y entregar información sobre nivelación de estudios y capacitación. 


\subsubsection{Articulación entre educación y empleo}

En Chile se promulgó el año 2008 la ley 20.267 que crea el Sistema Nacional de Certificación de Competencias Laborales (SNCCL), y que establece la existencia de la Comisión ChileValora, encargada de definir las políticas del sistema y cuya integración es de carácter tripartito, con participación de representantes de los trabajadores, empleadores y sector público (ministerios de Educación, Trabajo y Economía). ${ }^{5}$

ChileValora se basa en cuatro pilares fundamentales. La voluntariedad, la cual implica que tanto empleadores como trabajadores ingresan a este proceso en forma voluntaria para cualquier tipo de trabajo. La imparcialidad que se establece a través de la definición de procedimientos estandarizados para todos los centros de evaluación y certificación acreditados en el país. La orientación a la demanda, la cual implica el reconocimiento del sector que otorga la certificación. Por último, la libre competencia entre los que evalúan y certifican (ChileValora, 2011a; Chilecalifica, 2009b). En la misma perspectiva, desarrolla cuatro funciones conforme establece la ley: a) proponer políticas globales y velar por la transparencia, calidad y fe pública del sistema; b) crear y acreditar la nueva institucionalidad certificadora CECCL; c) levantar y acreditar competencias laborales a través de los sectores productivos, y d) informar y educar a los usuarios respecto del uso y beneficios del sistema (ChileValora, 2011b).

La relevancia estratégica de este sistema está dada por la posibilidad de detectar las demandas concretas del sector productivo y establecer los requisitos mínimos necesarios para el ejercicio de un oficio o trabajo determinado. El hecho de integrar tanto a los organismos de capacitación como a las entidades productivas permite actualizar permanentemente los requerimientos del medio,

5 Este organismo representa una nueva institucionalidad y es uno de los pilares fundamentales del Sistema de Formación Permanente destinado a "reconocer formalmente las competencias laborales de las personas, independientemente de la forma en que hayan sido adquiridas y de si tienen o no un título o grado académico otorgado por la enseñanza formal de conformidad a las disposiciones de la Ley No 18.962 Orgánica Constitucional de Enseñanza; así como favorecer las oportunidades de aprendizaje continuo de las personas, su reconocimiento y valorización" (Ley 20.267). 
garantizando la fe pública tanto de los organismos capacitadores acreditados como el desempeño cualificado de las personas. A lo anterior se suma la potestad de disponer de un registro público que contenga información pertinente asociada a la certificación de competencias en el ámbito nacional (ver Ley 20.267, Art.4).

Además, el MINEDUC a través de la Dirección de Educación Media ofrece la opción de la formación dual, que incorpora la asociación entre educación y trabajo, facilitando la empleabilidad y permitiendo, a través de la práctica, conocer mejor la especialidad elegida por los estudiantes. Para ello, se combinan los aprendizajes tanto en el establecimiento educacional como en la empresa. ${ }^{6}$

Otro de los pilares de la articulación entre educación y empleo es el Servicio Nacional de Capacitación y Empleo, SENCE, el cual realiza distintos tipos de actividades relacionadas con la capacitación en las empresas: apoya a los procesos de formación de trabajadores, define las gestiones destinadas a la capacitación, facilita los procesos de contratación, establece estándares asociados a competencias laborales, mantiene un registro de cursos oficialmente reconocidos y de organismos medios para la capacitación (OTIC) y apoya la alfabetización digital de trabajadores.

Finalmente, en cuanto a orientación vocacional, los establecimientos escolares suelen tener sicólogos especializados y orientadores cuya labor no trasciende al trabajo más personalizado con los jóvenes. Ello, dado que en la actualidad la relación entre orientadores y alumnos es de 1 a 500 a nivel nacional y en muchos colegios no se cuenta con este recurso humano (Espinoza, Castillo, Márquez, Mena y Álvarez, 2004).

Para aquellos jóvenes que prosiguen estudios superiores, el Consejo Nacional de Educación (CNED) provee información de todas las carreras que se imparten en el país mediante la base de datos y publicación INDICES, y el MINEDUC a través de la página web Futuro

6 Para acceder a la formación dual se requiere estar cursando tercer o cuarto año medio en un establecimiento técnico-profesional que se haya incorporado a la modalidad de formación dual. 
Laboral entrega información sobre las perspectivas laborales de 116 carreras profesionales y técnicas (CNED, 2009b).

En la perspectiva de la articulación entre educación y empleo, también el Programa Chilecalifica (2009c) entregaba antecedentes a los usuarios permitiéndoles relacionar educación y empleo mediante indicadores que daban cuenta de la realidad del mercado laboral y facilitaban la compatibilización de las aspiraciones con las oportunidades laborales existentes. Para ello se proveía información sobre las posibilidades de estudio y capacitación, así como aquella relacionada con las condiciones de funcionamiento de la economía y de las competencias requeridas por las empresas para desempeñarse en distintos puestos de trabajo (Chilecalifica, 2009d, 2009e). Con el término del Programa Chilecalifica esta línea desapareció del MINEDUC. En síntesis, respecto a los mecanismos asociados a las distintas estrategias para poner en práctica el aprendizaje permanente, cabe destacar que para la estrategia de articulación horizontal se establece como mecanismo más significativo la certificación y reconocimiento de estudios; para la estrategia de articulación vertical se asume como mecanismo prioritario la armonización curricular; y para la estrategia de articulación entre educación y empleo se plantea el mecanismo de certificación de competencias y el de orientación vocacional y profesional.

\subsection{Experiencias que podrían servir para la generación} de un sistema de información articulado que impulse el aprendizaje a lo largo de la vida

En aras de avanzar hacia un sistema de aprendizaje a lo largo de la vida, el Consejo de Innovación (CNIC, 2008) recomendó no solo consolidar, por un lado, un modelo de definición y certificación de competencias laborales que oriente los procesos formativos y, por otro, un conjunto de mecanismos de articulación del sistema, sino también fortalecer sistemas de información que permitan orientar y retroalimentar a los distintos actores del sistema de formación permanente.

En la actualidad, los ministerios y otras organizaciones afines proveen información útil para constituir un sistema de información 
integrado que fomente el aprendizaje a lo largo de la vida. Los antecedentes más relevante son provistos a través de la página web del MINEDUC, que contiene información general para los distintos niveles del sistema, incluyendo a los actores y al currículo, y el régimen de evaluación que incorpora aspectos curriculares, centros de recursos de aprendizaje, textos escolares, becas y resultados de las mediciones del SIMCE (MINEDUC, 2009a, 2009b).

Complementariamente, el MINEDUC entrega antecedentes sobre los programas de inglés, permite acceder al portal Enlaces que originalmente planteaba la interconexión virtual de estudiantes y profesores, y dispone del programa de subvenciones que se proponía instalar un computador en cada sala de clases de modo de no solo velar por la asistencia, sino también por el desarrollo y avance curricular de cada docente (MINEDUC, 2009b).

En la perspectiva de proveer información para la formación permanente, el MINEDUC ha generado igualmente un centro de atención ciudadana personalizado vía internet y telefónica que recibe consultas, facilita la realización de algunos trámites -como la solicitud de certificados, licencias y legalizaciones-, acoge sugerencias y recibe denuncias y reclamos.

En la misma línea del aprendizaje a lo largo de la vida, el MINEDUC está mandatado por ley para entregar información sobre el sistema de educación superior. Ésta se refiere a ayudas estudiantiles (becas y créditos), que permite postular a los programas de asistencia en línea, y al Observatorio del Empleo (Futuro Laboral), que ofrece información sobre empleabilidad y remuneraciones de los profesionales y técnicos (MINEDUC, 2009e).

Por intermedio del sitio web del MINEDUC, los estudiantes pueden acceder a la oferta académica, al directorio de instituciones, al ingreso especial y al reconocimiento de títulos. Asimismo, se informa de derechos y deberes de los estudiantes y tiene un enlace que vincula a la Ley Nacional de Servicio al Consumidor, institución que acoge reclamos de consumidores o beneficiarios de cualquier producto o servicio (MINEDUC, 2009d). 
Además del MINEDUC, existe en Chile un conjunto de otras entidades que están generando información educativa para la educación a lo largo de la vida. Otra experiencia que ya se ha mencionado es la del Consejo Nacional de Educación (CNED), que entrega información sobre el reconocimiento oficial de las instituciones de nivel terciario (CNED, 2009a) y publica anualmente INDICES (2009b), que contiene la oferta de programas de educación postsecundaria profesional y técnica de pre y posgrado, vacantes, duración, localización, modalidad, tipo de jornadas y costos de cada carrera y programa.

Otra de las instituciones que entrega datos para la educación a lo largo de la vida, en el nivel terciario, es la Comisión Nacional de Acreditación (CNA), que informa a la comunidad -estudiantes, padres, apoderados, orientadores vocacionales, y autoridades- sobre la calidad de las instituciones, carreras y programas.

También provee información relevante para los usuarios sobre las instituciones de educación superior el Consejo de Rectores de las Universidades Chilenas (CRUCH), que entrega antecedentes sobre los resultados y temarios de las pruebas nacionales de selección para el ingreso a la educación terciaria y un directorio de sus instituciones. Además, pone a disposición de la comunidad información anual de sus veinticinco universidades, por institución, área del conocimiento y región, sobre: vacantes de pregrado, matrícula de primer año y total de pregrado, matrícula total de posgrado, graduados a nivel de pre y posgrado (CRUCH, 2009).

Otras instituciones que generan información para la educación a lo largo de la vida son el Centro de Perfeccionamiento, Experimentación e Investigaciones Pedagógicas (CPEIP) que posee un Registro Público Nacional de Perfeccionamiento para profesores (CPEIP, 2009b); y el CONICYT (2009) que da cuenta de los distintos programas de becas para cursar estudios de posgrado tanto en Chile como en el extranjero.

Fuera del ámbito de la educación, el Ministerio del Trabajo a través del programa SENCE, como ya se ha señalado, entrega 
información para la educación permanente, informando en conjunto con la Fundación Chile sobre los estándares de desempeño de 600 competencias laborales.

En relación con la información sobre la capacitación en las empresas, el SENCE entrega antecedentes que proporciona la identificación de trabajadores debidamente capacitados para determinados puestos de trabajo, facilita los procesos de contratación y formación por competencias, así como la definición de estándares asociados a ellas. También, provee una base de datos con los cursos de capacitación. De igual manera, entrega datos para acceder a los organismos técnicos intermedios para la capacitación (OTIC) y provee información sobre los procesos de alfabetización digital y nivelación de estudios de los trabajadores como asimismo las opciones de formación técnica. Ha habido programas de capacitación entre los cuales se pueden citar los siguientes: Franquicia Tributaria para la Capacitación -que ha beneficiado a más de un millón de participantes-, Capacitación para Trabajadores Perceptores del Ingreso Mínimo Mensual, Becas Microempresas, Programa Especial de Capacitación para Microempresarios, Chile Emprende, Chilecalifica Línea Mype, Chilecalifica Línea NTIC, Becas Franquicia Tributaria (SENCE, 2009a, 2009b).

El Ministerio de Planificación, por su parte, dispone del sitio web del Programa FOSIS que da cuenta de todos los beneficios a los cuales pueden acceder beneficiarios de distintos subprogramas dirigidos a sectores vulnerables. Varios de esos programas tienen relación directa con la educación continua y la inserción en el mercado laboral (FOSIS, 2009a, 2009b).

De la misma manera, es interesante considerar a Chile Clic, un portal del Ministerio de Economía que registra información relativa a trámites y servicios para personas, empresas y organizaciones. En el sector educación, el sitio contiene un total de 234 fichas asociadas a distintos ámbitos tales como: alfabetización digital, ayudas estudiantiles, biblioteca, capacitación y educación de adultos, certificados, docentes y profesores, educación básica, educación media, educación parvularia, educación superior, posgrados y 
postítulos, estudiantes y profesores extranjeros, estudiantes y profesores chilenos en el extranjero (Ministerio de Economía, 2009).

Junto con lo anterior, existe una serie de otros sitios web que proveen información concerniente al aprendizaje a lo largo de la vida.

3.2.1. Iniciativas recientes para resguardar la calidad de la información pública

En la formación de capital humano avanzado se estableció un Sistema Nacional de Aseguramiento de la Calidad que, dentro de la diversidad, intenta asegurar estándares y criterios mínimos para garantizar la fe pública en el ejercicio profesional. El Sistema Nacional de Aseguramiento de la Calidad chileno incluye: el Consejo Nacional de Educación (CNED), que otorga autonomía a las nuevas instituciones después de un proceso de funcionamiento supervisado de a los menos seis años; la Comisión Nacional de Acreditación (CNA), que evalúa y acredita las instituciones y carreras, y también acredita y supervisa las agencias acreditadoras de carreras específicas.; el Servicio de Información sobre Educación Superior (SIES), y un Comité de coordinación integrado por el vicepresidente del CNED, el presidente de la CNA y el jefe de la División de Educación Superior del MINEDUC. Asimismo, se han establecido dentro del sistema escolar algunas instancias de evaluación vinculadas a la calidad, entre las que se puede mencionar el Sistema de Medición de Calidad de los Aprendizajes (SIMCE) que se aplica alternadamente cada año a los estudiantes de cuarto básico, octavo básico y segundo medio (SIMCE, 2009). También se aplica en el país la Prueba de Selección Universitaria (PSU) que regula el ingreso a la mayoría de las universidades del país (DEMRE, 2009). Y, por último, se encuentran los exámenes nacionales que se aplican obligatoriamente a los estudiantes de medicina y educación. A lo anterior, se suma la prueba de evaluación de desempeño que se aplica a los docentes de todo el país y que es administrada por el Centro de Perfeccionamiento, Experimentación e Investigaciones Pedagógicas (CPEIP, 2009a). 
Con relación al sistema escolar, el Congreso Nacional aprobó un proyecto de ley que crea una Superintendencia de Educación que, entre otras funciones, velará por la calidad de los servicios educativos. En forma complementaria, se creó un registro de asistencia técnica educativa (ATE) que agrupa a personas naturales y jurídicas con experiencia comprobada para apoyar la gestión de los establecimientos escolares y cuya labor es evaluada por los sostenedores.

Por su parte, la Comisión Nacional de Acreditación está iniciando el montaje de un sistema de información para la educación superior basado en indicadores de desempeño de las instituciones para informar al público, facilitar la toma de decisiones y resguardar la calidad del sistema. A lo anterior se suma la implementación del Servicio de Información sobre Educación Superior (SIES) por parte de la Divesup que pretende entregar antecedentes sobre el funcionamiento del sistema (MINEDUC, DESUP, 2009).

3.3. Recursos y medios para implementar un sistema de información de apoyo al aprendizaje a lo largo de la vida en Chile

Siguiendo con la lógica del modelo, en este apartado se da cuenta de los recursos y medios que existen en Chile que permitirían implementar un sistema de información de apoyo a la educación permanente. Específicamente, la descripción de los recursos y medios se ha organizado en relación con tres componentes: bases de información, páginas web, publicaciones y otros.

\subsubsection{Bases de información}

En Chile hay diferentes entidades del Estado que administran bases de información, entre las cuales se pueden mencionar el MINEDUC -a través de sus distintos organismos centralizados y descentralizados-, MINTRAB, Ministerio de Economía y MIDEPLAN.

Se observa que las bases de información no están suficientemente concatenadas y son alimentadas en forma independiente por cada una de las entidades que las administran. Esta situación, claramente, no 
favorece la búsqueda de información de respaldo para la toma de decisiones en relación al aprendizaje a lo largo de la vida.

\subsubsection{Páginas web}

Existe una cantidad significativa de páginas web nacionales que proveen información asociada con el aprendizaje a lo largo de la vida. Cada una de ellas da cuenta de temas específicos, entregando información detallada sobre los diversos aspectos considerados. Estas páginas están asociadas a algunos ministerios, los cuales disponen de numerosos portales vinculados al aprendizaje continuo. Además, no siempre están relacionadas entre sí y en ocasiones duplican información o bien entregan datos puntuales (referidos a un mismo indicador) que no coinciden. Cabe reseñar que hay páginas que contienen bastante información, tal como acontece con el sitio del MINEDUC y el SENCE. En cuanto al diseño y alcances de sitios web, Chile presenta en la actualidad sitios de alta calidad pero que no se articulan debidamente.

\subsubsection{Publicaciones y otros}

En el país se publican en periódicos de alta circulación antecedentes sobre las opciones educacionales (INDICES) y sobre resultados académicos (SIMCE) que facilitan las decisiones de los usuarios. En algunos casos, las publicaciones impresas son replicadas en formato electrónico y las publicaciones electrónicas son editadas en versiones impresas. También se divulga información estadística como la registrada en los Anuarios Estadísticos del CRUCH y folletería de programas de apoyo que generan MIDEPLAN y el MINTRAB. Asimismo, el MINEDUC ofrece algunos servicios restringidos de consulta personalizada por vía telefónica.

Resulta evidente que Chile está en línea con las tendencias de los países desarrollados en el sentido de potenciar las publicaciones electrónicas por sobre las publicaciones impresas, como una herramienta para orientar y facilitar la toma de decisiones de los distintos tipos de usuarios, tanto en materia educativa como laboral. No obstante, aparece a la zaga en cuanto al desarrollo de una atención más personalizada basada en los centros de llamada, los que eventualmente podrían ser subsidiados por el Estado. 


\section{Problemas actuales para generar un sistema de información en Chile que fomente el aprendizaje a lo largo de la vida y requerimientos de los usuarios}

En este apartado se han considerado dos aspectos. En primer lugar, una descripción de los principales problemas que limitan un adecuado funcionamiento de los subsistemas existentes. Y, en segundo término, una síntesis de las necesidades y demandas detectadas para superar las debilidades del servicio de información de apoyo a la educación permanente.

\subsection{Análisis de las diversas fuentes de información disponibles asociadas a la educación permanente}

$\mathrm{Al} \mathrm{revisar} \mathrm{las} \mathrm{diferentes} \mathrm{fuentes} \mathrm{de} \mathrm{información} \mathrm{disponibles} \mathrm{y} \mathrm{las} \mathrm{bases}$ de datos que se relacionan con la educación a lo largo de la vida, se puede constatar que existe un conjunto de debilidades que pueden incidir en la optimización del uso y socialización de los recursos. Entre las debilidades detectadas se pueden observar tres grupos: uno referido a los sistemas en su conjunto, otro vinculado con aspectos técnicos, principalmente referidos a los datos, y un tercer grupo asociado a los actores (proveedores, usuarios y policy makers).

En relación con el primer grupo de debilidades se constata que no existe en la actualidad una plataforma única e integrada que recoja información relevante asociada al aprendizaje a lo largo de la vida. Ello en parte se explica por la inexistencia de una política articulada destinada a fortalecer la educación continua. Se denota una carencia de articulación suficiente entre las bases de datos que administran las distintas entidades, lo cual está condicionado en parte por una cultura que no apunta a la integración ni a la interrelación de instituciones proveedoras de datos. Como consecuencia de lo anterior se aprecia una dispersión de la información sin tener una visión global que beneficiaría a los usuarios.

En lo que concierne a las debilidades de carácter técnico, se verifican incoherencias en las definiciones de los datos, como asimismo reiteraciones y diferentes categorizaciones para una misma información, o bien incompatibilidades del formato. Ello conduce a 
que la información en algunos casos sea de poca calidad y, por ende, no confiable. Por otra parte, no hay una adecuada actualización, con lo cual la información va perdiendo su vigencia y utilidad. A lo anterior se suma la falta de rigurosidad en el manejo y construcción de series históricas y un limitado seguimiento para detectar errores.

En cuanto al tercer grupo de debilidades que se vincula a los actores, se puede señalar en referencia a los proveedores que las instituciones, en general, presentan reluctancia y lentitud para entregar los datos. A ello se suma la rotación del personal encargado del diseño y mantenimiento de las bases de datos. En relación a los usuarios, falta identificar las necesidades reales para distintos grupos objetivo y muchas veces se verifica que hay un desconocimiento de los recursos existentes por parte de éstos. Respecto a los policy makers, se puede señalar que si bien muchas veces existen los datos, estos no están suficientemente trabajados mediante un análisis técnico que redunde en indicadores de mayor complejidad y que facilite la toma de decisiones.

\subsection{Demandas y necesidades de los usuarios}

A partir del análisis de las distintas fuentes de información asociadas al aprendizaje a lo largo de la vida, se visualiza la necesidad de disponer de un sistema de información integrado, amigable e interactivo, para lo cual se requiere lograr una mayor coordinación entre las entidades nacionales que proveen información, y tener la posibilidad de cruzar información de diferentes fuentes de modo de cumplir cabalmente con la función de informar a la sociedad y a las distintas poblaciones.

Para disponer de un sistema de calidad se requiere que la información tenga las siguientes características: homologable, coherente, consistente, oportuna, confiable, válida y actualizada, rasgos descritos detalladamente en el marco conceptual.

Un sistema con estas características permitirá entregar información diferenciada a distintos usuarios en función de sus demandas, como asimismo realizar estudios y formular políticas con el respaldo técnico necesario. 


\section{Recomendaciones para implementar un sistema de información integrado}

En los párrafos siguientes se entrega un conjunto de recomendaciones sobre la base del diagnóstico previamente desarrollado, con la idea de generar un sistema integrado de información de apoyo para el aprendizaje a lo largo de la vida y se proveen sugerencias para su implementación.

\subsection{Aspectos a considerar para generar un sistema de información integrado}

Las sugerencias que aquí se presentan se han estructurado de acuerdo a los problemas actuales, demandas y necesidades de los usuarios teniendo en consideración para ello las experiencias exitosas de otros países. La elaboración de una plataforma única que integre datos de distintas fuentes y que permita a usuarios heterogéneos ubicar la información requerida para demandas diversas puede ser una solución adecuada para superar la dispersión existente en la actualidad. Para ello se propone implementar bases relacionales con datos concatenados a través de variables comunes, como por ejemplo el rol único nacional que se asigna al nacer a cada chileno.

Para enfrentar las debilidades de carácter técnico, es necesario establecer una modalidad de trabajo que garantice mediante procesos de compilación y verificación que la calidad de la información entregada satisfaga verdaderamente las demandas, para lo cual debe contarse con equipos, recursos humanos y procedimientos adecuados.

Para superar las debilidades asociadas con los actores, es indispensable establecer procedimientos para agilizar y garantizar la entrega de información confiable y oportuna por parte de las instituciones proveedoras. De igual forma, se podría externalizar los servicios de diseño y mantenimiento, de modo de evitar los problemas y vacíos que genera la rotación de personal. También, se propone generar encuestas periódicas a los distintos tipos de usuarios para asegurarse que la información entregada les resulte relevante y útil. Asimismo, se sugiere que se establezcan procedimientos para procesar los datos, de tal suerte que satisfagan las necesidades de expertos, por un lado, y de público de menor preparación, por otro. 
Para estructurar un sistema de información integrado, se propone organizar los antecedentes considerando cuatro dimensiones: i) etapas de la trayectoria educativo-laboral; ii) requerimientos de información de diferentes usuarios; iii) usuarios propiamente tal; y iv) organismos proveedores de información.

En relación a las etapas de la trayectoria educativo-laboral, esta dimensión incluye los niveles educativos de preescolar, básica, media (científico-humanista y técnico-profesional), superior y de adultos, así como la educación no formal a través de la capacitación y el perfeccionamiento y los datos sobre ocupación y empleo.

En lo que respecta a los requerimientos de los usuarios se consideran los datos sobre establecimientos educacionales y entidades capacitadoras, opciones de programas, cursos y carreras, indicadores de calidad, localización de la oferta educativa o de formación, costos y opciones de financiamiento, formas y procesos de postulación, convalidaciones, certificaciones, planes y programas de estudio, opciones de orientación vocacional y profesional, y tendencias del mercado laboral.

Entre los usuarios preferentes hacia los cuales están dirigidos estos sistemas se encuentran estudiantes e interesados, padres y apoderados, sostenedores, técnicos y expertos, servicios de orientación, organismos certificadores, y empleadores

En cuanto a los organismos proveedores de información, se propone establecer las responsabilidades de los ministerios incluyendo educación, trabajo, planificación y economía. Ello, en el bien entendido de que cada una de estas entidades dispone de un conjunto de organismos, servicios y programas que aportan datos en relación con la información que demandan los usuarios.

Como se muestra en la tabla $\mathrm{n} .^{\circ} 2$, lo más relevante respecto a la propuesta es la articulación de los sitios existentes mediante una plataforma que permita concatenar a través de enlaces los antecedentes que se requieren para los distintos usuarios en las diferentes etapas de la trayectoria educativa y laboral. 


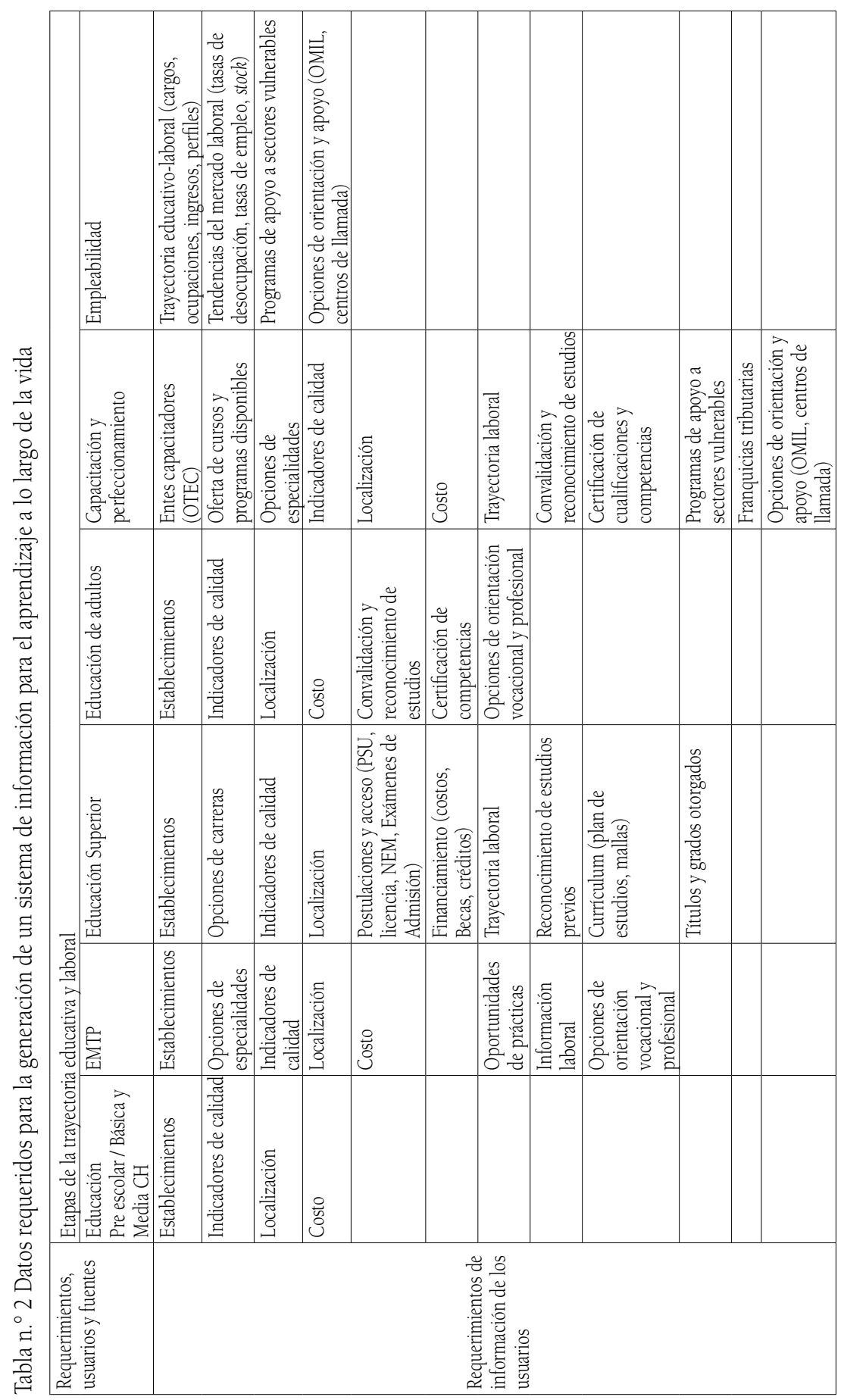


156 EXPERIENCIAS Y ASPECTOS A CONSIDERAR PARA LA IMPLEMENTACIÓN DE UN SISTEMA DE INFORMACIÓN DE APOYO PARA EL APRENDIZAJE A LO LARGO DE LA VIDA EN CHILE - O. Espinoza, L.E. González

\begin{tabular}{|c|c|c|c|c|c|c|c|c|}
\hline 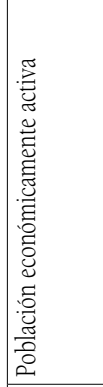 & 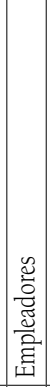 & 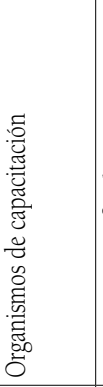 & 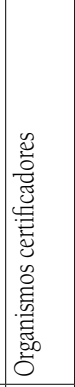 & 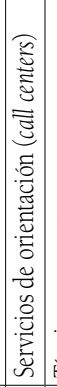 & 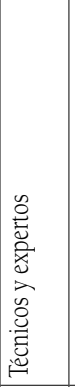 & & 局 & \\
\hline 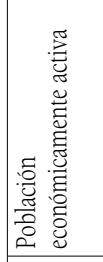 & 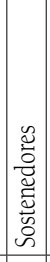 & 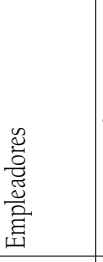 & 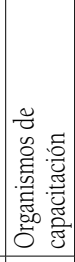 & 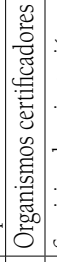 & 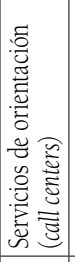 & 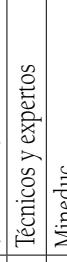 & | & 遆 \\
\hline 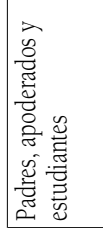 & 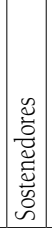 & 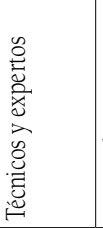 & 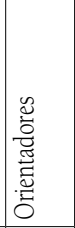 & & & & $\begin{array}{l}0 \\
\text { 总 } \\
\text { 竞 }\end{array}$ & \\
\hline 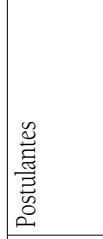 & 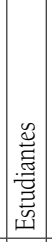 & 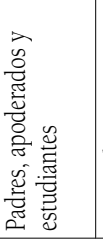 & 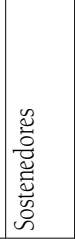 & 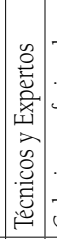 & 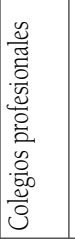 & 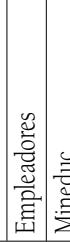 & 总 & 芯 \\
\hline 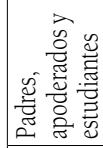 & 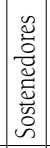 & 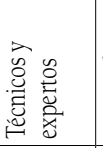 & 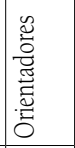 & 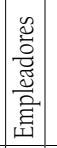 & & & 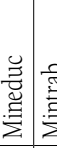 & \\
\hline 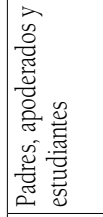 & 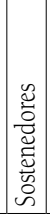 & 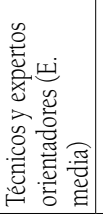 & & & & & 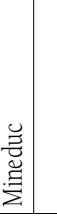 & \\
\hline & & 恚 & & & & & \multicolumn{2}{|c|}{ 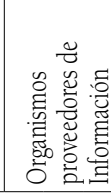 } \\
\hline
\end{tabular}




\subsection{Recomendaciones para la implementación de un sistema de información integrado}

A partir de los problemas previamente delimitados y de la propuesta para implementar un sistema de información integrado para la educación permanente, se formulan a continuación algunas recomendaciones para su puesta en marcha.

Para la implementación de un sistema de información integrado se deben considerar tres dimensiones: montaje y mantenimiento de la plataforma, aspectos técnicos de la información e interacción con proveedores y usuarios involucrados.

La lógica indica que montaje y mantenimiento de la plataforma debería estar alojada en el Ministerio de Educación, para lo cual se requeriría un sistema de apoyo técnico y administrativo constante. La construcción de dicha plataforma debiera lograrse mediante el concurso de profesionales idóneos y altamente capacitados tanto en la parte tecnológica como en la referida a la sistematización de información y construcción de indicadores.

En los aspectos técnicos es necesario establecer un control y monitoreo de la información. Para ello debiera existir una unidad que tenga, entre otras, las siguientes funciones:

i) solicitar información a las distintas instancias con autoridad para exigir el envío de datos;

ii) definir variables e indicadores relevantes y consistentes para distintos ministerios, organismos y servicios;

iii) revisar la consistencia y confiabilidad de los datos que entregan los proveedores, incluyendo el análisis de series históricas;

iv) socializar la información de manera amigable, pensando que se está apuntando a un público amplio y heterogéneo, con distintas necesidades; $y$

v) garantizar que exista información oportuna y actualizada de los diferentes organismos y programas.

Es fundamental generar mecanismos de consultas periódicas que permitan detectar las demandas de usuarios y proveedores. Se recomienda que un grupo de especialistas recabe sistemáticamente 
opiniones de los actores a través de distintos mecanismos, tales como, encuestas, grupos focales, entrevistas grupales, etc. Por otra parte, se requiere de especialistas en el ámbito comunicacional que diseñen formatos de presentación de datos amigables, de fácil acceso y manipulación para un público heterogéneo. De igual forma, se sugiere que se generen informes técnicos especializados para satisfacer las demandas de los expertos y de los policy makers.

En términos de la interacción con los usuarios, no basta con tener buenos sistemas de información sino que además deben existir los enlaces adecuados para que los antecedentes sean accesibles a quienes los demandan. En tal sentido, adquieren preponderancia los servicios de orientación y de atención más personalizada, en particular para atender a las poblaciones más vulnerables del país. Ello requiere de un esfuerzo importante que implica, por una parte, mejorar el servicio de orientación vocacional que se provee en los establecimientos escolares y, por otra, generar servicios telefónicos o centros de llamadas que den la posibilidad de una atención más focalizada a quienes lo necesitan. El montaje de centros de llamada como el existente en Inglaterra con un subsidio del Estado constituye una buena opción para ser replicada en Chile. Se propone optimizar la atención a los usuarios, ampliando los centros de llamadas (call centers) y las líneas de atención al usuario como lo han hecho algunos países de la OCDE. De este modo, se dispondría de una herramienta más innovadora sin aumentar significativamente el gasto público, al reducir el tiempo destinado a las entrevistas presenciales, que podría utilizarse en aquellos casos de mayor complejidad. Asimismo, se sugiere ampliar y flexibilizar el horario de atención de estos centros, con el fin de facilitar un mayor acceso a este servicio de consejería profesional. Para optimizar el servicio, se sugiere establecer flujos de derivación de los usuarios mediante "filtros" asociados a los temas de consulta y sus niveles de complejidad.

Para enfrentar los problemas de falta de coordinación que se observa entre las distintas instituciones generadoras de información relativas al aprendizaje a lo largo de la vida, se sugiere instaurar una entidad interministerial con plena autonomía, que articule las necesidades y demandas de instituciones y de diferentes usuarios en materia de información. Ello implica desarrollar procesos asociados 
con la detección de necesidades, selección, recolección, actualización, validación, sistematización, procesamiento, y difusión de información confiable, válida y oportuna.

En concordancia con lo anterior, se propone montar un sistema nacional de cualificaciones, considerando todos los niveles del sistema educacional tanto formal como no formal y lo referido al ámbito de la empleabilidad con una mirada prospectiva.

\section{Referencias bibliográficas}

Araneda, H. (2006), "Hacia un sistema de aprendizaje a lo largo de la vida: oportunidad del sistema de certificación de competencias laborales". Serie En Foco n. ${ }^{\circ}$ 75. Expansiva, Santiago de Chile.

Behringer, F. y Coles, M. (2003). The Role of National Qualifications Systems in Promoting Lifelong Learning. Towards an Understanding of the Mechanisms that Link Qualifications and Lifelong Learning. OCDE Education Working Paper n. ${ }^{\circ} 3$.

Bermejo, B. (2006). La formación a lo largo de la vida: Exigencias sociolaborales y desarrollo personal.

Disponible en http://tecnologiaedu.us.es/formaytrabajo/Documentos/ lin5ber.pdf

CEDEFOP (Sellin, B. 2002), Scenarios and strategies for vocational education and lifelong learning in Europe, summary of findings and conclusions of the joint CEDEFOP/ETF project (1998-2002), Luxembourg, OOPEC, Panorama series.

Chilecalifica (2009a) ¿Qué ofrece la educación de personas jóvenes y adultas? Disponible en http://www.chilecalifica.cl/califica/showNoti.do?noticia $=2855 \&$ barra $=2 \&$ pagina $=$ bajada-noticia-frame.jsp

Chilecalifica (2009b). Sistema Nacional de Certificación de Competencias. Un paso trascendental en la instalación del Sistema de Formación Permanente.

Disponible enn http://www.chilecalifica.cl/califica/showNoti. do?noticia $=2742 \&$ barra $=2 \&$ pagina $=/$ bajada-noticia-frame.jsp

Chilecalifica (2009c). Sistema de Formación Permanente.

Disponible en http://www.chilecalifica.cl/prc/n-2713-Presentacion.pdf

Chilecalifica (2009d). Capacitación Laboral.

Disponible enhttp://www.chilecalifica.cl/califica/showNoti. do?noticia=364\&barra=2\&pagina=/bajada-noticia-frame.jsp 
160 EXPERIENCIAS Y ASPECTOS A CONSIDERAR PARA LA IMPLEMENTACIÓN DE UN SISTEMA DE INFORMACIÓN DE APOYO PARA EL APRENDIZAJE A LO LARGO DE LA VIDA EN CHILE - O. Espinoza, L.E. González

Chilecalifica (2009e). Información y Orientación.

Disponible enn http://www.chilecalifica.cl/califica/showCuerpo.do?c uerpo $=292 \&$ aplic $=1 \&$ barra $=1 \&$ pagina $=$ bajada-cuerpo-frame.jsp

ChileValora (2011a). Estructura ChileValora.

Disponible enhttp://www.chilevalora.cl/prontus_chilevalora/site/ artic/20100407/pags/20100407174233.html

ChileValora (2011b). Funciones de la Comisión.

Disponible enn http://www.chilevalora.cl/prontus_chilevalora/site/ artic/20100414/pags/20100414002439.html

CINDA (2000). Acreditación de programas, reconocimiento de títulos e integración. Experiencia en países latinoamericanos y europeos. Santiago: CINDA.

CNIC (2008). Hacia una Estrategia Nacional de Innovación para la Competitividad. Volumen 2. Santiago: CNIC.

CONICYT (2009). Programa de Capital Humano Avanzado.

Disponible en http://www.conicyt.cl/573/propertyvalue-1756.html

CPEIP (2009a). Evaluación del Desempeño Profesional Docente.

Disponible en http://www.cpeip.cl/website/index2.php?id_ portal $=1$ \&id_contenido $=85$

CPEIP (2009b). Registro Público Nacional de Perfeccionamiento.

Disponible en http://www.cpeip.cl/website/index2.php?id_ portal=1\&id_contenido $=76$

CRUCH (2009). Anuarios Estadísticos.

Disponible en http://www.cruch.cl/anuarios_estadisticos.html

CRUCH (2007). Guía Práctica para la Instalación del SCT-Chile. Sistema de Créditos Académicos Transferibles.

Disponible en http://www.sct-chile.cl/descargas.htm

CNED (2009a). Acerca del CSE.

Disponible en http://www.cse.cl/public/Secciones/seccionacercade/ acerca_de_cse_que_es_cse.aspx

CNED (2009b). Estadísticas y Bases de Datos. INDICES.

Disponible en http://www.cse.cl/public/Secciones/SeccionEstadisticas/ Estadisticas_Bases.aspx

Delors, J., Mufti, A., Amagi, I., Carneiro, R., Chung, F., Geremek, B., Gorham, W., Kornhauser, A., Manley, M., Quero, P., Savané, M.A., Singh, K., Stavenhagen, R., Won Suhr, M., Nanzhao, Z. (1996). Learning: The Treasure Within. Report to UNESCO of the International Commission on Education for the Twenty-first Century. Paris, Unesco Publishing. 
DEMRE (2009). La PSU. Disponible en http://www.demre.cl/psu.htm.

Espinoza, O., Castillo, D., Márquez, P., Mena, F. y Álvarez, E. (2004). Estudio de Interés Vocacional y Laboral en Jóvenes de Enseñanza Media. Santiago: Chilecalifica-PIIE.

Espinoza, O., González, L.E., Castillo, D. y Loyola, J. (2011). Factores familiares asociados a la deserción escolar en Chile: un estudio de caso. En Revista de Ciencias Sociales, Universidad de Zulia (en imprenta).

Espinoza, O., González, L.E. y Uribe, D. (2011). La Probabilidad de Terminar la Educación Media y de Acceder a la Educación Superior en Chile: Análisis Estadístico de Modelos. En CINDA, El Proceso de Transición entre educación media y superior (305-321). Santiago, CINDA.

Fosis (2009a). ¿Qué es el Fondo de Solidaridad e Inversión Social - Fosis? Disponible en http://www.fosis.cl/opensite_55.asp

Fosis (2009b). Programas.

Disponible en http://www.fosis.cl/opensite_20081013164128.asp

González, L. E. y Ayarza, H. (Eds.) (1998). Reconocimiento y convalidación de estudios superiores y títulos profesionales en América Latina y el Caribe. Santiago: CINDA.

González, L. E. (2000). Acreditación en la educación superior chilena. En Ayarza, H. (Editor). Acreditación de Programas, reconocimiento de títulos e integración. Experiencia en países latinoamericanos y europeos (pp.89-114). Santiago: CINDA.

Latorre, C.L., González, L.E. y Espinoza, O. (2009). Equidad en Educación Superior: Evaluación de las Políticas Públicas de la Concertación. Santiago, Editorial Catalonia/ Fundación Equitas. 245 pp.

Ley 20.267 (2008). Crea el Sistema Nacional de Certificación de Competencias Laborales y Perfecciona el Estatuto de Capacitación y Empleo. Disponible en http://www.bcn.cl/leyes/pdf/original/272829.pdf

MINEDUC (2009). El Sistema Educacional Chileno.

Disponible en http://www.Mineduc.cl/index.php?id_portal=16\&id_ seccion $=1479$ \&id_contenido $=2759$

MINEDUC (2009a). Becas y créditos para tu educaron superior.

Disponible en En http://www.becasycreditos.cl/

MINEDUC (2009b). Contenidos.

Disponible en http://www.mineduc.cl/index0.php?id_portal=1\#

MINEDUC (2009c). Estadísticas e información oficial del sistema escolar en Chile. Disponible en http://w3app.mineduc.cl/DedPublico/Inicio 
162 EXPERIENCIAS Y ASPECTOS A CONSIDERAR PARA LA IMPLEMENTACIÓN DE UN SISTEMA DE INFORMACIÓN DE APOYO PARA EL APRENDIZAJE A LO LARGO DE LA VIDA EN CHILE - O. Espinoza, L.E. González

MINEDUC (2009d). Educación Superior.

Disponible en http://www.educacionsuperiorchile.cl/

MINEDUC (2009e). Futuro Laboral.cl. Carreras y Mundo del Trabajo. En http://www.futurolaboral.cl/FuturoLaboral/index.html

MINEDUC (2009f).Exámenes de validación de estudios o "exámenes libres" Disponible en http://www.mineduc.cl/index.php?id_portal=19\&id_ seccion $=458 \&$ \&id_contenido $=275$

MINEDUC, DESUP (2009). Sistema de Información de la Educación Superior, SIES.

Disponible en http://www.mece2.com/portal/content/view/73/207/ lang,spanish/

Ministerio de Economía (2009). Chile Clic Guía de Servicios del Estado.

Disponible en http://www.chileclic.gob.cl

Molitor, M. (2005). "Movilidad estudiantil y cambio curricular. La experiencia europea”. En CINDA, Movilidad Estudiantil (pp.157-170). Santiago: CINDA.

OCDE (2003). Career Guidance and Public Policy: Bridging the Gap. Paris: OCDE.

OCDE (2007). "Qualifications Systems: Bridges to Lifelong Learning". OCDE. Abstract disponible en: http://www.oecd.org/document/53/0,3343, $\mathrm{fr}_{-}$ 2649_34859051_38465013_1_1_1_1,00.html

Pacheco, P., Brunner, J.J., Elacqua, G. y Salazar, F. (2005). Nuevas Competencias, Exigencias, Profesionales y Life Long Learning.

Disponible en http://mt.educarchile.cl/mt/jjbrunner/archives/ Plataforma\%20Life\%20Long\%20Learning.htm

Prieto, J. P. y Mujica, C. (2007). Sistema de Créditos Transferibles y Carga de Trabajo de los Estudiantes en las Universidades del Consejo de Rectores. En Revista Calidad de la Educación n. ${ }^{2} 26,1^{\circ}$ Semestre año 2007, pp.293-306. Consejo Superior de Educación, Santiago de Chile.

Sence (2009a). Quienes Somos. Objetivos Estratégicos.

Disponible en http://www.sence.gob.cl/Portal.Base/Web/VerContenido. aspx?ID=576\&IDI=187

Sence (2009b). Quienes Somos. Programas Sence 2008.

Disponible en http://www.sence.gob.cl/Portal.Base/Web/VerContenido. aspx?ID=578\&IDI=190

SIMCE (2009). ¿Qué es el SIMCE?

Disponible en http://www.simce.cl/index.php?id=288\&no_cache=1 
Zúñiga, M. (2005). Movilidad estudiantil y articulación horizontal entre universidades.

Artículo presentado a Seminario Internacional "Intercambio Estudiantil y Cambio Curricular", celebrado en la Universidad Nacional de Cuyo Mendoza, 11 y 12 de agosto del 2005.

Recibido: 8/04/2011

Aceptado: 18/05/2011 